\title{
Recent IceCube Results from Searches for Transient Neutrino Sources
}

\author{
A. Homeier for the IceCube collaboration* \\ Universitaet Bonn \\ E-mail: ahomeier@icecube.wisc.edu
}

\begin{abstract}
IceCube, a cubic kilometer neutrino detector located in glacial ice at the South Pole, has recently reached a sensitivity below the TeV-PeV neutrino flux that is predicted by some models of gammaray bursts (GRBs) if those are responsible for the observed extragalactic cosmic-ray flux and are accelerating protons. No excess neutrinos over background have been observed, and an upper limit on the flux of energetic neutrinos associated with GRBs is now at least a factor of 3.7 below the predictions of fireball models. A complementary online neutrino multiplet event selection allows IceCube to trigger Swift and a network of optical telescopes, which can then identify possible electromagnetic counterparts. This allows to probe for the connection between GRBs, $\mathrm{SNe}$ and relativistic jets in SNe. Results from the GRB search and a first limit on relativistic jets in $\mathrm{SNe}$ are presented.
\end{abstract}

Gamma-Ray Bursts 2012 Conference -GRB2012,

May 07-11, 2012

Munich, Germany

\footnotetext{
* Speaker.
} 


\section{Introduction}

Gamma-ray bursts (GRBs) have long been proposed [1,2] as the acceleration sites of the highest energy cosmic rays due to an aggregate power density in photons nearly matching that in cosmic rays. This hypothesis is difficult to verify experimentally - the transient nature of the sources makes their direct correlation to cosmic rays or identifying hadronic signatures in gamma ray spectra challenging. However, interactions of high-energy protons with the intense radiation in the environment of the burst should produce pions which then decay into neutrinos [10]:

$$
\begin{array}{r}
\pi^{+} \rightarrow \mu^{+}+v_{\mu} \\
\mu^{+} \rightarrow e^{+}+\overline{v_{\mu}}+v_{e}
\end{array}
$$

These neutrinos are then expected to arrive at Earth with energies of approximately $100 \mathrm{TeV}$ [10] with fluxes at a level that can be detected with large terrestrial detectors such as IceCube. Observation of this neutrino flux would then provide unambiguous evidence for hadronic acceleration in GRBs, and strongly suggest that GRBs are responsible for some fraction of the ultra high-energy cosmic ray flux.

The observed GRB-SN connection [4] gives rise to the idea that GRBs and SNe might have the jet signature in common and a certain fraction of core-collapse SNe might host soft relativistic jets. Such soft relativistic jets would become stalled in the outer layers of the progenitor star, leading to essentially full absorption of the electromagnetic radiation and at the same time an efficient production of high-energy neutrinos $[5,6]$.

In an effort to search for neutrinos from these transient objects two strategies were devised. We present the most recent search in IceCube for neutrinos in temporal and directional coincidence with reported GRBs. Complementary to this offline search, neutrinos produced in soft relativistic $\mathrm{SN}$ jets can be detected in real time by IceCube and can be used to trigger follow-up observations [8].

\section{IceCube Detector}

The IceCube neutrino telescope [9] has been under construction at the geographic South Pole since 2004 and was completed in the Antarctic summer of 2010/11. It is capable of detecting high energy neutrinos with energies of $\mathscr{O}(100) \mathrm{GeV}$ and is most sensitive to muon neutrinos with energies in the $\mathrm{TeV}$ range and above. High-energy muon neutrinos undergoing charged current interactions in the ice or the underlying rock produce muons in neutrino-nucleon interactions. The muon travels in a direction close to that of the neutrino and emits Cherenkov light. The deep ultra clear Antarctic ice is instrumented with light sensors thus forming a Cherenkov particle detector. Since its completion it comprises a volume of $1 \mathrm{~km}^{3}$ with 5160 digital optical modules (DOMs) attached to 86 vertical strings at a depth of $1450 \mathrm{~m}$ to $2450 \mathrm{~m}$. Each DOM consists of a $25 \mathrm{~cm}$ diameter Hamamatsu photomultiplier tube (PMT) and supporting hardware inside a glass pressure sphere. Here we present the offline analysis of data taken with the 40-string detector configuration (2008/04/05 to 2009/05/20) and the 59-string configuration (2009/05/20 to 2010/05/25) as well as the analysis of online IceCube data taken in conjunction with the optical follow-up program from 
2008/12/16 to 2009/12/31. In the following the deployment stages will be referred to as IC40 and IC59.

\section{Search for neutrinos in coincidence with observed GRBs}

We conducted two analyses of the IceCube data, both searching for neutrino emission coincident with satellite detections of GRBs, as reported through the GRB Coordinates Network [9]. Both used stacked catalogs (combining the event sample for all observed GRBs) and employed the same methods as in the 40-string search [7]. In a model-dependent analysis, we searched specifically for the predicted neutrino flux during the period of gamma-ray emission using the spectral prediction of [3] (Fig. 1). In a complementary model-independent analysis, we searched more generically for neutrinos with a wider range of energies and an expanding time window centered on the burst time and expanding in 1-second increments in both directions to encompass a 2-day period centered on the GRB time. Both analyses shared a catalog of 215 northern-hemisphere GRBs during the IC40 and 59 string runs, with an additional 85 southern-hemisphere bursts included in the 59-string model-independent search, and obtained similar results when evaluating the

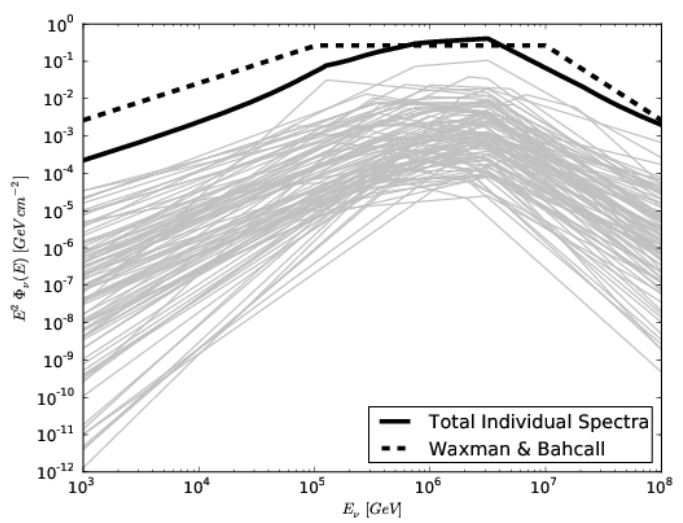

Figure 1: Predicted neutrino spectra of the GRBs used in the triggered offline analysis. The thin lines represent the individual bursts while the solid thick line represents the sum of all bursts. Finally, the dashed line shows the [10] prediction normalized to the number of GRBs observed. same models. No signal-like events were observed in either analysis.

Using this non-observation we set stringent limits on the neutrino flux from GRBs [16]. These limits exclude all tested models [10, 11, 3, 12] with their parameters and uncertainties (fig. 3). For the Waxman-Bahcall (WB) model [10] our limit is 0.27 times the predicted flux. Our results very strongly constrain the parameter space of fireball models. Increasing the bulk Lorentz factor leads to an increase of the proton energy threshold for pion production in the observer frame thereby reducing the neutrino flux. Another possibility is to decrease the energy deposited in protons in comparison to electrons $\varepsilon_{p} / \varepsilon_{e}$ decreasing the density of high energetic protons and as such the neutrino flux. Our current constraints on the fireball parameters are presented in fig. 2, and the parameter space will be probed even more stringently in future years. Models based on the fireball phenomenology normalize the predicted neutrino flux to the measured cosmic ray flux assuming that all of the high energy extragalactic cosmic rays are coming solely from GRBs. Either GRBs are not the only sources of cosmic rays or efficiency of neutrino production is much lower than has been predicted. 


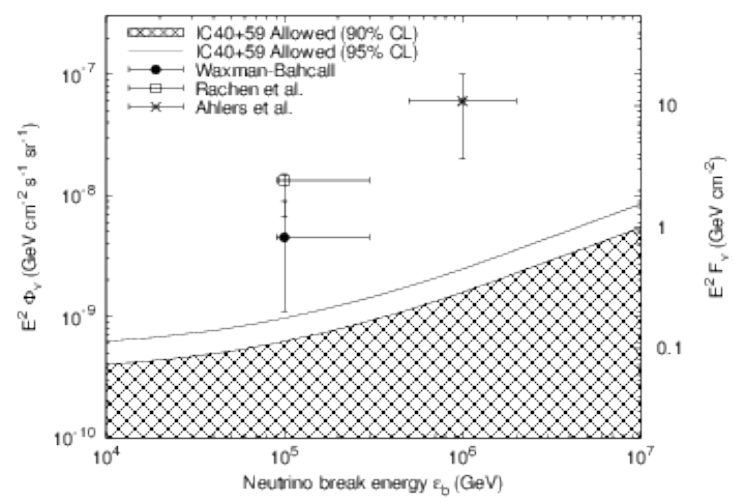

Figure 2: Compatibility of cosmic-ray flux based models with observations. Shown are the allowed values of neutrino flux vs. the neutrino break energy $\left(\varepsilon_{b}\right)$ in comparison to model predictions with estimated uncertainties. Data were taken from the modelindependent analysis from the time window corresponding to the median duration of the GRBs in our catalog $(|\Delta t|=28$ seconds).

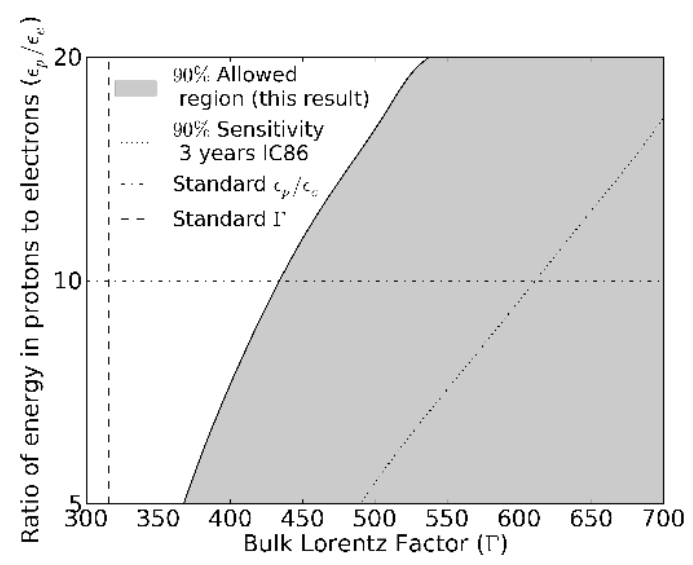

Figure 3: Constraints on fireball parameters. The shaded region, based on the result of the model-dependent analysis, shows the values of GRB energy in protons and the average fireball bulk Lorentz factor for modeled fireballs allowed by this result at the $90 \%$ confidence level.

\section{Search for soft jets in core-collapse SNe with IceCube Optical Follow-Up Program}

The analysis presented here was designed to be sensitive to neutrino production in soft relativistic SN jets. To suppress the background of atmospheric neutrinos, which we cannot distinguish from the soft SN neutrino spectrum, we require the online detection of at least two neutrino events in IceCube within $100 \mathrm{~s}$ and an angular difference between their two reconstructed directions of $\Delta \Psi \leq 4^{\circ}$ or less. A doublet is not significant by itself, but may become significant when the optical information is added.

The IceCube multiplet alerts are forwarded to the Robotic Optical Transient Search Experiment (ROTSE) [13], which observes the corresponding region of the night sky within seconds. Follow-up observations are performed regularly for 3 weeks.

Due to the online processing latency of several hours we focused on the SN light curve detection in the ROTSE data. Image subtraction followed by a detection algorithm to identify transient source candidates are applied to find an optical counterpart.

\begin{tabular}{|l|cc|cc|c|}
\hline & \multicolumn{2}{|c|}{ Doublets } & \multicolumn{2}{c|}{ Triplets } & SN \\
& IC40 & IC59 & IC40 & IC59 & \\
\hline measured & 15 & 19 & 0 & 0 & 0 \\
\hline expected & 8.55 & 15.66 & 0.0028 & 0.0040 & 0.074 \\
\hline
\end{tabular}

Table 1: Measured and Expected Multiplets 
Here the results from the analysis of data taken in the period of 2008/12/16 to 2009/12/31 are presented. Table 1 shows the number of detected and expected doublets, triplets and optical SN counterparts. The number of doublets shows a small excess, which corresponds to a $2.1 \sigma$ effect and is thus not statistically significant. The expected number of randomly coincident $\mathrm{SN}$ detections, $N_{\mathrm{SN}}^{b g}=0.074$, is based on an assumed core-collapse $\mathrm{SN}$ rate of 1 per year within a sphere of radius $10 \mathrm{Mpc}$, i.e. 2.4 $10^{-4} \mathrm{y}^{-1} \mathrm{Mpc}^{-3}$, and a Gaussian absolute magnitude distribution [14]. No optical SN counterpart was found in the data.

We obtain the confidence level for different

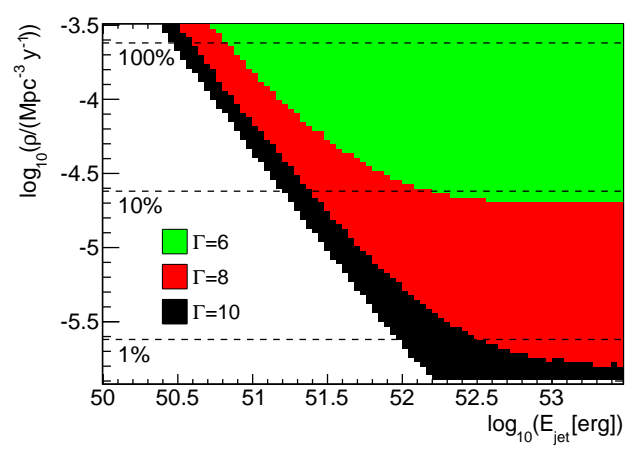

Figure 4: Limits on the choked jet SN model [6] for different boost Lorentz factors $\Gamma$ as a function of the rate of $\mathrm{SNe}$ with jets $\rho$ and the jet energy $E_{\text {jet }}$ (colored regions are excluded at $90 \% \mathrm{CL}$ ). Horizontal dashed lines indicate a fraction of $\mathrm{SNe}$ with jets of $100 \%, 10 \%$ or $1 \%$. combinations of SN model parameters [6] by using a pre-defined test statistic based on a likelihood function. The limit is calculated for the jet boost Lorentz factors $\Gamma=6,8,10$ as a function of the rate of SNe with jets $\rho$ and the jet energy $E_{\text {jet }}$. Systematic errors related to the simulated neutrino sensitivity and the SN sensitivity are included in the limit calculation. The $90 \%$ confidence regions for each $\Gamma$-value are displayed in the $E_{\text {jet }}-\rho$-plane in Fig. 4. A sub-population of SN with typical values of $\Gamma=10$ and $E_{\text {jet }}=3 \cdot 10^{51} \mathrm{erg}$ does not exceed $4.2 \%$ (at 90\% confidence). This is the first limit on CCSN jets using neutrino information [17].

Because of the successful operation of the optical follow-up program with ROTSE, the program was extended in August 2010 to the Palomar Transient Factory [15] and to an X-ray follow-up by the Swift satellite in February 2011.

\section{References}

[1] E. Waxman, Cosmological Gamma-Ray Bursts and the Highest Energy Cosmic Rays, Phys. Rev. Lett. 75 (1995) 386

[2] M. Vietri, The acceleration of ultra-high energy cosmic rays in gamma-ray bursts, ApJ 453:883 (November 1995)

[3] D. Guetta et al., Neutrinos from individual gamma-ray bursts in the BATSE catalog, Astropart. Phys. 20 (2004) 429

[4] S.Woosley and J. Bloom, The Supernova - Gamma-Ray Burst Connection, Ann. Rev. Astron. Astrophys. 44 (2006) 507

[5] S. Razzaque et al., High Energy Neutrinos from a Slow Jet Model of Core Collapse Supernovae, Mod. Phys. Lett. A20 (2005) 2351

[6] S. Ando and J. F. Beacom, Revealing the Supernova - Gamma-Ray Burst Connection with TeV Neutrinos, Phys. Rev. Lett. 95 (2005) 061103

[7] R. Abbasi et al., Constraints on high-energy neutrino emission from SN 2008D, A\&A 527 (2011a) A28

[8] M. Kowalski and A. Mohr, Detecting Neutrino Transients with Optical Follow-up Observations, Astropart.Phys. 27 (2007) 533 
[9] A. Achterberg et al., First Year Performance of the IceCube Neutrino Telescope, Astropart. Phys. 26 (2006) 155

[10] E. Waxman, Astrophysical sources of high energy neutrino, Nucl.Phys. B Proc.Suppl. 118 (2003) 353

[11] J. P. Rachen and P. Meszaros., Cosmic Rays and Neutrinos from Gamma Ray Bursts, 4th Hunstville Symposium, volume 428 of American Institute of Physics Conference Series 776-780 (1998)

[12] M. Ahlers, M. C. Gonzalez-Garcia, and F. Halzen, GRBs on probation: Testing the UHE CR paradigm with IceCube., Astropart. Phys. 35:87 (2011) 94

[13] C. W. Akerlof et al., The ROTSE-III Robotic Telescope System, PASP 115 (2003) 132

[14] D. Richardson et al., ABSOLUTE MAGNITUDE DISTRIBUTIONS AND LIGHT CURVES OF STRIPPED-ENVELOPE SUPERNOVAE, AJ 131 (2006) 2233

[15] A. Rau et al., Exploring the Optical Transient Sky with the Palomar Transient Factory, PASP 121 (2009) 1334

[16] R. Abbasi et al. (IceCube Collaboration), An Absence of Neutrinos Associated with Cosmic-Ray Acceleration in Gamma-Ray Bursts, Nat. 484 (2012) 351 [arXiv: 1204 . 4219]

[17] R. Abbasi et al. (IceCube Collaboration), Searching for Soft Relativistic Jets in Core-collapse Supernovae with the IceCube Optical Follow-up Program, A\&A 539 (2012) A60s [arXiv:1111.7030] 